\title{
EDITORIAL
}

\section{Eradicating infectious diseases in 2010}

\author{
Disease eradication programmes are often slower than expected. Several local and \\ regional eradication programmes had targets for 2010, but progress towards these goals \\ has been variable.
}

December 2009 marked the thirtieth anniversary of the certification of smallpox eradication. This triumph encouraged thinking about the eradication of other diseases, not only those of humans but also those of livestock, which can be of great economic importance. Although no other infectious disease has been eradicated since 1979, several local and global eradication programmes have deadlines for 2010 .

The good news is that in 2010 a second infectious disease, rinderpest, is likely to be declared eradicated. This viral disease of ruminants, primarily cattle, is related to human measles and has plagued herds for centuries, causing large losses of cattle and other livestock. The Global Rinderpest Eradication Programme was started in 1994, and the last case of rinderpest was detected in 2001. The next human disease to be eradicated will most probably be Guinea worm disease. Although the original eradication target of 2009 has been missed, progress has been encouraging: the number of cases of Guinea worm disease reported worldwide from January to October 2009 dropped by $31 \%$ from 2008 levels to approximately 3,000 cases, which is a $99.9 \%$ decrease from the incidence rate when the eradication programme started in 1986.

Unfortunately, two regional efforts, targeting yaws in Southeast Asia and measles in Europe, are unlikely to meet their targets of eradication in 2010. Yaws, a disfiguring disease caused by Treponema pallidum subsp. pertenue, was eradicated in India in 2004 but remains endemic in Indonesia and East Timor, although the number of infections is decreasing. A new goal for eradication has been set for 2012. The eradication of measles in Europe has been set back by a drop in the vaccination rate. A well-publicized scare in the United Kingdom about the potential side effects of the MMR (measles, mumps and rubella) vaccine and the difficulty in reaching certain members of the European population have caused vaccination rates to fall below the $95 \%$ level that is thought to be required to block transmission. Nonetheless, endemic measles could be eradicated in Europe in the near future, if the vaccination rates can be raised in those countries where the disease is still present.
The most elusive and well-documented eradication effort is the polio eradication programme. The eradication campaign was started in 1988 by the World Health Assembly, with the goal of eradicating polio by 2000 . After a large initial decrease in the number of cases worldwide, with a low of 453 cases in 2001, the number has fluctuated around 1,500 cases for the past few years. Political factors and armed conflicts in endemic areas, as well as religious opposition, have created gaps in the vaccination coverage that have allowed disease transmission to continue, making it difficult to predict when eradication will be achieved.

Failure to complete eradication programmes is costly. A previous effort to eradicate yaws that was started in the 1950s was never completed, and the disease has disfigured hundreds of thousands of individuals since then, although the incidence rate has remained low compared with the rate before the eradication effort started. However, this is not the case for malaria. When the malaria eradication effort of the 1950s and 1960s was halted, the disease soon reached unprecedented levels. Similarly, a disease as contagious as polio is likely to surge if the eradication effort is halted, causing many people to become paralyzed and costing developing countries large sums in lost productivity.

Conversely, successful eradication programmes bring great rewards. The human benefits can be enormous, and a substantial financial burden can be removed from endemic regions. For example, in the United States the last endogenous case of smallpox occurred in 1949, and vaccination stopped in 1971. The United States contributed approximately US\$32 million to the worldwide eradication effort and "has subsequently recouped its investment every few weeks, in avoided costs, disease and deaths" (REF. 1), according to Donald Hopkins, Vice President for Health Programs at The Carter Center. It is therefore imperative to see these eradication programmes through. Too much has been invested to let these diseases return when the finish line is in sight.

. Hopkins, D. The allure of eradication. Global Health [online] http:// www.globalhealthmagazine.com/top_stories/the allure of eradication (2009). 